\title{
Les marbres : noblesse de la matière, heureuses « curiosités » de la nature
}

\section{Sophie Mouquin}

\section{(2) OpenEdition}

1 Journals

Édition électronique

URL : http://journals.openedition.org/artefact/523

DOI : $10.4000 /$ artefact.523

ISSN : 2606-9245

Éditeur :

Association Artefact. Techniques histoire et sciences humaines, Presses universitaires du Midi

\section{Édition imprimée}

Date de publication : 1 octobre 2016

Pagination : 347-359

ISBN : 978-2-7535-5174-9

ISSN : 2273-0753

\section{Référence électronique}

Sophie Mouquin, «Les marbres : noblesse de la matière, heureuses « curiosités » de la nature », Artefact [En ligne], 4 | 2016, mis en ligne le 07 juillet 2017, consulté le 15 septembre 2020. URL : http:// journals.openedition.org/artefact/523

\section{(c) (1) $\odot$}

Artefact, Techniques, histoire et sciences humaines est mise à disposition selon les termes de la Licence Creative Commons Attribution - Pas d'Utilisation Commerciale - Pas de Modification 4.0 International. 


\section{Les marbres : noblesse de la matière, heureuses " curiosités » de la nature ${ }^{1}$}

Sophie MoUQuIN²

De toutes les matières que l'art puise dans la nature, le marbre, entendu dans son acception artistique, c'est-à-dire celle d'une " pierre dure qui reçoit un beau poli » est l'une de celles qui suscite les dialogues les plus féconds entre sciences dures et sciences humaines, entre géologie et histoire de l'art. Matière reine de la statuaire et matière très appréciée pour l'architecture et les décors, elle fut pendant longtemps délaissée des historiens de l'art, à l'exception des spécialistes de sculpture. L'étude de son approvisionnement et de sa mise en œuvre, notamment dans les grands décors et les collections de la période moderne, est relativement récente. Alors que de nombreux travaux sur la typologie des marbres et l'histoire de leur extraction et de leur utilisation existent pour la période antique ${ }^{3}$, il faut attendre la fin du $x^{e}$ siècle pour que des connaisseurs comme Jacques Dubarry de Lassale $^{4}$ et des historiens de l'art de la période moderne envisagent ce champ de la recherche, notamment dans ses applications françaises. L'histoire du marbre, de la carrière à sa mise en œuvre retient l'attention d'ouvrages récents, sans cependant que les aspects techniques, notamment ceux de l'extraction, ne soient véritablement étudiés hormis dans la connaissance, lacunaire, que livrent les archives. À la suite de ceux de célèbres historiens ou historiens de l'art italiens, les travaux de Geneviève Bresc-Bautier et Hélène Du Mesnil qui révélèrent l'histoire de l'approvisionnement sous l'Ancien Régime, envisageant notamment les réseaux marchands ${ }^{5}$, ou encore ceux de Jean-Louis Bonnet et de Pascal Julien qui s'intéressèrent à l'histoire des carrières pyrénéennes et languedociennes ${ }^{6}$, démontrèrent la pertinence de ce champ d'études pour l'histoire de l'art. Nos propres travaux, dans les mêmes années, s'intéressèrent aux marbriers qui travaillaient pour la couronne de France, et à l'administration des marbres qui favorisa la fourniture en marbre notamment sous les règnes de Louis XIV et de Louis XV7. Ces approches, historiques, fondées sur des documents d'archives, ont permis d'identifier les carrières, de révéler les difficultés matérielles d'extraction ou d'acheminement, de mettre à jour les réseaux marchands et le système administratif et économique, de découvrir la personnalité des principaux acteurs et de raconter une « histoire du marbre ", celle de l'extraordinaire aventure de l'approvisionnement en marbre en France sous l'Ancien Régime. Plus qu'en termes techniques, l'étude du marbre par les historiens de l'art est donc 
envisagée dans ses aspects économiques et pratiques, sans cependant que l'analyse de sa mise en œuvre ne soit oubliée : les études d'Alexandre Cojannot pour les débuts du règne de Louis XIV ou encore celles de Muriel Barbier pour les règnes de Louis XV et de Louis XVI, ont renouvelé la connaissance de l'utilisation du marbre dans l'architecture et le décor intérieur ${ }^{8}$.

Mais l'histoire de l'art est aussi histoire du goût et en ce domaine, les marbres et les pierres "dures » tiennent une place particulière. La terminologie est d'une savoureuse imprécision. L'amateur et le curieux considèrent en effet, sans les différencier, des pierres « ornementales » ou " marbres » qui relèvent cependant de typologies géologiques bien différentes. L'histoire de l'art et l'histoire du goût n'ont pas la précision scientifique de l'histoire des techniques. Ainsi, parmi les marbres ou " pierres dures", les amateurs rangent le jaspe, la serpentine, le porphyre, les agates, les cornalines et autres pierres fines (mais non précieuses) et non uniquement les " calcaires ou dolomies métamorphiques ", seuls marbres des géologues9. L'appréciation de ces marbres, pierres dures ou pierres fines, n'est pas nouvelle. Depuis l'Antiquité, les amateurs goûtent la beauté de

\section{Les figures du marbre}

"Pierre dure qui reçoit un beau poli, qui est difficile et longue à tailler ${ }^{11} »$ le marbre fut, dès l'Antiquité, apprécié pour les veines qui le composent et dessinent parfois d'élégantes figures. Le pavé du Tibur de Vopiscus, composé de "mosaïques ces créations de la nature que l'homme se plaît à graver, monter ou enchâsser. À la Renaissance, les pierres dures apparaissent dans les cabinets de curiosités, comme objet de délectation du curieux, mais aussi dans l'architecture et le mobilier, disposées en cabochons, telles des pierres précieuses qu'elles ne sont pourtant pas $^{10}$. Cette apparition des pierres dures dans le décor architectural et mobilier prépare d'une certaine manière, le goût, en France, pour une architecture et un décor où le marbre tient une place que jusqu'à présent seule l'Italie lui avait donnée. Les grands décors de Louis XIV favorisent le développement d'une véritable "politique royale du marbre » pour reprendre l'heureuse expression de Geneviève Bresc-Bautier.

Il est impossible d'envisager, en quelques pages, toute cette histoire du marbre. Considérant les "marbres» dans leur acception esthétique des $\mathrm{XVII}^{\mathrm{e}}$ et $\mathrm{XVIII}^{\mathrm{e}}$ siècles, sans les restreindre aux seuls marbres géologiques, nous n'envisagerons ici, ni l'histoire des techniques, ni l'histoire de leur approvisionnement, ni celle de leur mise en œuvre, mais celle de leur appréciation par les amateurs et les savants à un moment où le dialogue entre science et curiosité devint d'une remarquable fécondité. animées par de nouvelles figures », c'està-dire sans doute de marbres veinés, faisait les délices de Stace ${ }^{12}$. Ce goût pour les dessins que l'amateur peut se plaire à reconnaître dans les replis de la matière se double d'une croyance, développée 
notamment par Pline, en la vie même du marbre qui cacherait en son sein une figure que l'artiste libère. Le récit relaté dans l'Histoire naturelle du "grand prodige » survenu dans les carrières de Paros où « les mineurs ayant détaché de la carrière, par le moyen des coins, un grand bloc de marbre, y trouvèrent une empreinte naturelle représentant Silène ${ }^{13}$ ", traverse toute l'histoire de la pensée et se retrouve notamment chez Plotin ou Michel-Ange. Fénelon se réapproprie également la pensée plinienne dans sa Démonstration de l'existence et des attributs de Dieu:

"Qui trouveroit dans une île déserte et inconnue à tous les hommes une belle statue de marbre, diroit aussitôt : sans doute il y a eu ici autrefois des hommes : je reconnois la main d'un habile sculpteur : j'admire avec quelle délicatesse il a su proportionner tous les membres de ce corps, pour leur donner tant de beauté, de grâce, de majesté, de vie, de tendresse, de mouvement et d'action. Que répondroit cet homme si quelqu'un s'avisoit de lui dire : non, un sculpteur ne fit jamais cette statue. Elle est faite, il est vrai, selon le goût le plus exquis, et dans les règles de la perfection; mais c'est le hasard tout seul qui l'a faite. Parmi tant de morceaux de marbre, il y en a eu un qui s'est formé ainsi de lui-même; les pluies et les vents l'ont détaché de la montagne; un orage très violent l'a jeté tout droit sur ce piédestal, qui s'étoit préparé de lui-même dans cette place. C'est un Apollon parfait comme celui du Belvédère : c'est une Vénus qui égale celle de Médicis : c'est un Hercule qui ressemble à celui de Farnèse. Vous croiriez, il est vrai, que cette figure marche, qu'elle vit, qu'elle pense, et qu'elle va parler : mais elle ne doit rien à l'art; et c'est un coup aveugle du hasard, qui l'a si bien finie et placée $^{14}$. »

Au début du XVIII ${ }^{\mathrm{e}}$ siècle, Gottfried Wilhelm Leibniz, dans les Nouveaux essais sur l'entendement humain, qu'il rédige en 1703 mais qui ne paraissent qu'en 1765, fait référence à la même idée :

« Je me suis servi aussi de la comparaison d'une pierre de marbre qui a des veines, plutôt que d'une pierre de marbre toute unie, ou des tablettes vides, c'est-à-dire de ce qui s'appelle tabula rasa chez les philosophes. Car si l'âme ressemblait à ces tablettes vides, les vérités seraient en nous comme la figure d'Hercule est dans un marbre, quand ce marbre est tout à fait indifférent à recevoir ou cette figure ou quelque autre. Mais s'il y avait des veines dans la pierre qui marquassent la figure d'Hercule préférablement à d'autres figures, cette pierre y serait plus déterminée, et Hercule y serait comme inné en quelque façon, quoi qu'il faudrait du travail pour découvrir ces veines, et pour les nettoyer par la polissure, en retranchant ce qui les empêche de paraître ${ }^{15}$. »

D'après Gilles Deleuze l'image des veines $\mathrm{du}$ marbre s'applique chez Leibniz sous deux conditions différentes : " tantôt les veines sont les replis de la matière qui entourent les vivants pris dans la masse, si bien que le carreau 
de marbre est comme un lac ondoyant plein de poissons. Tantôt les veines sont les idées innées de l'âme, comme les figures pliées ou les statues en puissance prises dans le bloc de marbre ${ }^{16}$ ». Ce lac ondoyant plein de poissons, cette métaphore maritime ou aqueuse pour le marbre, a fait l'objet de plusieurs études, notamment par Fabio Barry, dans un article qui a fait date et qui démontrait que certains décors antiques ou médiévaux avaient habilement utilisé les veines du marbre pour transformer sols ou parois en mers, vagues, en espaces animés et vibrants ${ }^{17}$.

Les veines du marbre peuvent ainsi constituer des figures, naturelles ou humaines, des paysages et des personnages, soit naturellement, soit parce que l'artisan travaille habilement la matière. Raniero Gnoli, auteur bien connu des spécialistes du marbre, considérait que " les veines et les taches naturelles du marbre peuvent être combinées de manière à simuler ou à suggérer des images variées. C'est surtout vrai dans les décorations pariétales constituées de plaques de marbre découpées dans un même bloc et disposées en livre ouvert $^{18} »$. Mais plus encore que la capacité de l'homme à savamment disposer les veines du marbre pour suggérer des images, ce sont les marbres qui dessinent naturellement des paysages ou des figures qui font les délices des amateurs. Les pietre paesine, ces " marbres » des environs de Florence, sont particulièrement appréciés aux $\mathrm{XVII}^{\mathrm{e}}$ et $\mathrm{XVIII}{ }^{\mathrm{e}}$ siècles, et font, encore aujourd'hui, le bonheur de certains curieux et de certains hommes de lettres qui célèbrent ces « pierres-paysages où la fantaisie de la nature imite et invente des ports, des maisons, des flottes en bataille et des visions de Giotto, percent tout à coup, sous le désordre minéral, la main de l'homme et son effort, et surgit lentement un dessin : un reste de porte, un arc, une tour effondrée, un rempart ${ }^{19}$ ». Ce goût pour la fantaisie naturelle que Roger Caillois et André Beton considéraient comme une langue ou une écriture ${ }^{20}$, cette célébration de l'inventivité de la création se retrouve en Orient dans l'appréciation par les lettrés chinois des "pierres de rêve ", ces « rochers » qu'ils interprètent comme des paysages et des poèmes, ces « os du ciel et de la terre ", microcosmes et macrocosmes de l'univers qui garnissent leurs cabinets ${ }^{21}$. Ces « images » naturelles, ces «bizarreries de la nature » expliquent l'emploi du marbre dans le domaine de l'architecture et du décor, mais également l'engouement pour la collection d'échantillons les plus rares, ces naturalia qui garnissaient les cabinets de curiosités des plus grands amateurs notamment à la Renaissance, mais aussi aux $\mathrm{XvII}^{\mathrm{e}}$ et $\mathrm{xVIII}^{\mathrm{e}}$ siècles $^{22}$. Le sujet mériterait un ouvrage et fut d'ailleurs plusieurs fois étudié : il est évident que l'appréciation des veines du marbre, au xviII siècle, est héritière d'un goût pour les pierres dures et fines qui « jouent l'art et la nature » pour reprendre l'heureuse expression employée par Léopold d'Autriche pour décrire, en 1628, un cabinet recouvert "d'agates, de cornalines, de calcédoines et de jaspes agencées avec des tableautins peints à l'huile ${ }^{23}$ ». Les " images » que contiennent les marbres, les paysages que dessinent les veines des pierres dures, résultent, comme le résume Jurgis Baltrušaitis dans ses célèbres Essais sur la légende des formes, «d'une même spéculation sur l'art de la Nature et la nature de 
l'Art, où pierre et vie se superposent et se confondent dans le débordement des fantaisies baroques ${ }^{24}$ ».

Si l'intérêt pour les figures du marbre apparaît dès l'Antiquité, c'est surtout au $\mathrm{XVII}^{\mathrm{e}}$ siècle que l'appréciation esthétique se double d'une recherche scientifique. La formation des images naturelles que contiennent les pierres fait l'objet de plusieurs théories. Dans son Dictionnaire raisonné universel d'Histoire naturelle, Jacques Christophe Valmont de Bomare rappelait que plusieurs "physiciens" éminents, comme "Pline, Aldrovande, Kircher, Boccone, Agricola, Ferrante Imperati ", s'étaient intéressé aux « jeux de la nature » que constituent les marbres veinés et les pierres figurées ${ }^{25}$. En 1665, Athanasius Kircher, dans son Mundus Subterraneus, donnait quatre hypothèses : «1) le hasard;2) une disposition de la substance terrestre à recevoir une empreinte, qui est ensuite pétrifiée; 3) la pétrification accidentelle d'un corps ou d'un objet; 4) une disposition divine particulière, réalisée par les influences angéliques et naturelles ${ }^{26} »$. Tout au long des $\mathrm{XVII}^{\mathrm{e}}$ et XVIII ${ }^{\mathrm{e}}$ siècles l'explication de la formation des « figures » des marbres reste approximative. Les scientifiques ne parviennent pas à comprendre l'origine des "singularités " naturelles que constituent « les tortuosités de certaines racines, les nœuds de quelques bois, les figures d'autres pierres, les desseins que présentent les marbres de Florence $\&$ les Agathes de divers lieux, où les différentes figures qu$^{\prime}$ offre la glace ${ }^{27}$ ». Benoît de Maillet, Dezallier d'Argenville, Buffon ont des explications divergentes : en ce milieu du $\mathrm{xvIII}^{\mathrm{e}}$ siècle la rigueur scientifique, si elle s'affirme peu à peu, n'en reste pas moins approximative.
Pour Benoît de Maillet (1636-1738), dont le Telliamed, publié seulement en 1748, influença la génération des naturalistes de la seconde moitié du xviII ${ }^{\mathrm{e}}$ siècle, les « bigarrures » des marbres étaient de deux sortes : la première " est l'effet de certaines ondes, qui se rencontrent principalement dans les marbres de couleurs d'agathe, dans les rougeâtres, dans les verds et dans ceux qui approchent de ces couleurs "; tandis que la bigarrure " accidentelle » consiste en " certaines rayes, ordinairement blanches ou jaunes, qui se trouvent dans ces mêmes marbres, $\&$ dans plusieurs carrières de pierres ${ }^{28}$ ». Pour le comte de Buffon (1707-1788), qui commence à composer son Histoire naturelle dès 1749 , il faut distinguer

« la partie du fond qui d'ordinaire est de couleur uniforme, d'avec les autres parties qui sont par taches ou par veines, souvent de couleur différentes; les veines traversent le fond et sont rarement coupées par d'autres veines, parce qu'elles sont d'une formation plus nouvelles que le fond et qu'elles n'ont fait que remplir les fentes occasionnées par le dessèchement de cette matière $\mathrm{du}$ fond ${ }^{29}$ ».

Buffon, chez qui la description l'emporte, est l'un des rares à distinguer veines et taches : " toutes les taches sont irrégulièrement terminées et comme frangées à leur circonférence, tandis que les veines sont au contraire sans dentelures ni franges et simplement tranchées des deux côtés dans leur longueur ${ }^{30}$ ». En 1755, Antoine-Joseph Dezallier d'Argenville (1680-1755), plus téméraire, se risque à expliquer la genèse des veines : 
«Ces égouts en tombant sur les matrices, devraient former des taches rondes de la même manière que font ordinairement les gouttes d'eau : les élévations de matière déjà congelée qu'elles trouvent dans les matrices les font agir autrement, elles les obligent de couler en long dans les parties basses, \& de former

\section{Le nuancier de la nature}

$\mathrm{Si}$ les veines et figures des marbres sont une ode à la nature et à la création et expliquent que les "pierres dures", aient fasciné les amateurs et intéressé les savants - même si ces derniers ne parvinrent pas véritablement à percer les mystères de leur formation -, au XVIII ${ }^{\mathrm{e}}$ siècle, le goût pour les marbres est aussi la célébration de l'heureuse diversité du nuancier polychrome de la nature (illustrations 22 et 23, cahier couleur). Pour Antoine-Joseph Dezallier d'Argenville comme pour l'auteur de l'article sur les Marbres dans le Journal économique de juin $1759^{32}$, la couleur des marbres est le résultat d'un hasard plus ou moins heureux :

« Les marbres sont formés, suivant un auteur [Célapin] d'une matière pure, concrète, amassée par coagulation, c'est la même que celle des pierres, mais elle est plus épurée. Divers égouts tombent du ciel d'une carrière sur les matrices des pierres, apportent avec eux différents sels, les uns passant contre une mine de cuivre ou de vitriol, chacune en serpentant des veines de la couleur qu'elles apportent : elles tracent ainsi en se mêlant ensemble des figures confuses, entremêlées les unes avec les autres, \& des espèces de compartiments tels qu'on les remarque dans la bigarrure des marbres $^{31}$.» dont des taches vertes sur la pierre; les autres venant $d$ 'une mine de fer forment la couleur jaune; il en est de même des autres couleurs ${ }^{33}$. »

Cette idée d'une "pénétration "se retrouve chez de nombreux savants comme Benoit de Maillet selon lequel les couleurs des marbres étaient liées à la terre sur laquelle ils avaient été formés. Si le " rouge du marbre de Savaresse [Seravezza] est si beau ", c'est, affirme-t-il, parce que "sur les montagnes des environs il se rencontre une terre d'un rouge si vif, que les canaux par où les eaux des pluies coulent de ces montagnes à la mer, semblent teints de sang ${ }^{34}$ ». Sont ainsi expliquées " toutes les autres couleurs, dont les carrières de cette nature sont variées dans tous les pays différents du globe $^{35} »$. De même, il considère que les marbres « bigarrés ", sont " formés d'une matière boueuse \& aisée à se déjeter ", mais « frappés de l'air, du soleil \& de la gelée, ils s'étaient entr'ouverts, \& que recevant dans leurs fentes les eaux des pluies, \& celles de la mer qui les surmontait encore, ils avaient contracté ces bigar- 
rures, suivant les terres et les limons dont ces eaux étaient chargées, cette matière qu'on peut regarder comme une espèce de colle ou de ciment, ayant servi à réunir différentes pièces ou écailles, dans lesquelles leur superficie s'était déjà partagée $^{36}$ ». Comme pour les veines dont les dessins étaient appréciés des amateurs, les savants ne parvinrent pas à donner d'explication véritablement satisfaisante à la formation de la couleur des marbres.

Pourtant l'intérêt pour la polychromie des pierres constitue un élément clé de la définition même du marbre. En 1698, Nicolas Lémery considérait qu'il existait « trois espèces générales de marbre, un blanc, un noir \& un de diverses couleurs $^{37}$ ». Ce type de classification resta longtemps en vigueur chez les naturalistes. Le comte de Borch distinguait, dans sa Minéralogie sicilienne publiée en 1780, les marbres à une couleur, des panachés et des brèches, c'est-à-dire relativement « à la nature et à la formation de chacun d'eux », mais en réalité aussi de leur élément coloré ${ }^{38}$. Même GeorgesLouis Leclerc de Buffon accordait à la couleur une place décisive dans sa classification, considérant même qu'il " y a de ces pierres qui sont presque aussi dures, aussi denses et d'un grain aussi fin que les marbres, et auxquelles néanmoins on ne donne pas le nom de marbres parce qu'elles sont sans couleur décidée ou plutôt sans diversité de couleur ${ }^{39}$ ». Le célèbre naturaliste reprenait aussi l'idée, désormais communément admise, d'un suc pétrifiant comme élément indispensable à la solidification des madrépores et coquilles, qui, «fortement imprégné des couleurs du fer ou d'autres minéraux », donne leurs teintes aux marbres. Buffon prenait cependant le soin de préciser que très peu de marbres sont monochromes, à l'exception du noir et du blanc, et que " du mélange de ces diverses couleurs, il résulte une infinité de nuances différentes ». Mais le savant apportait une précision très intéressante : la possibilité

«d'augmenter par l'art la vivacité et l'intensité des couleurs que les marbres ont reçues [sic] de la nature; il suffit pour cela de les chauffer [...] en les polissant à chaud, et ces nouvelles nuances de couleur, acquises par un moyen si simple, ne laissent pas d'être permanentes et ne s'altèrent ni ne changent par le refroidissement ni par le temps : elles sont durables parce qu'elles sont profondes, et que la masse entière $\mathrm{du}$ marbre prend par cette grande chaleur ce surcroît de couleurs qu'elle conserve toujours ${ }^{40} »$.

Les premiers essais de coloration artificielle des marbres étaient vraisemblablement anciens. Les spécialistes d'art antique, grec surtout, et les restaurateurs connaissent et étudient depuis longtemps ce phénomène, y compris dans des aspects très techniques qui ne sont pas de notre sujet, pas plus que la création, encore relativement méconnue pour la période moderne, de la production de marbres factices, nous attachant à ce que les ouvrages du XvIII ${ }^{\mathrm{e}}$ siècle révèlent de la connaissance de la coloration des marbres ${ }^{41}$. Pline s'en fait l'écho dans son Histoire Naturelle (livre xxxv), précisant que l'invention remontait au temps de l'empereur Claude et que " sous Néron, on imagina de mélanger les marbres simples » et que l'on parvint ainsi à enrichir « de taches de pourpre » 
le Synnadique, suppléant ainsi à la nature et créant " des marbres tels que les désiraient nos caprices ${ }^{42}$ ». Dans la seconde moitié du xvIII ${ }^{\mathrm{e}}$ siècle, Athanase Kircher $^{43}$ et Nicolas Lémery ${ }^{44}$ livrèrent des recettes qui permettaient d'obtenir des effets colorés. En 1730, 1'Académie royale des sciences publia un "Mémoire sur la teinture \& la dissolution de plusieurs espèces de pierres » dans lequel M. du Fay [de la Faye] retraçait diverses expériences sur les "moyens de faire pénétrer dans l'agathe, dans le marbre \& dans plusieurs autres pierres dures, différentes espèces de couleurs ${ }^{45} »$. De toutes les pierres dures, le marbre, blanc surtout, y était présenté comme l'une des plus faciles à colorer. L'auteur du mémoire donne maints détails sur les différentes colorations artificielles et se vantait d'avoir obtenu un bleu pur, tout en reconnaissant qu'il était impossible d'obtenir un noir parfait. D'après Michel-Jean de Borch, la coloration des marbres, grâce à des teintes végétales, était couramment employée en Sicile par les marbriers pour " corriger les défauts des marbres du pays » ou pour " tromper les étrangers peu connaisseurs en leur vendant un marbre coloré pour un marbre naturel ${ }^{46} »$. Ce type d'artifice semble avoir été assez largement employé : Alex-Frederik Cronstedt, chimiste averti, dénonçait la pratique, visiblement répandue en Italie, selon laquelle " à défaut d'originaux, on prend des espèces semblables à leur place \& on colore aussi des sortes de marbres blancs $^{47} »$.

Il est certain que les marbriers et les lapidaires savaient réaliser des mastics colorés susceptibles de cacher les imperfections d'un marbre ou encore les cas- sures qui pouvaient survenir lors de la taille ou la pose. Les veines tant appréciées des amateurs et des savants sont autant de fragilités de ces pierres dures, mais néanmoins délicates. Mais certains allèrent visiblement plus loin, colorant véritablement le marbre, soit par fourberie, soit pour apporter à un marbre blanc notamment une touche de polychromie. Piganiol de La Force atteste ainsi que les joues de la figure de Pudicita, placée dans la galerie des Glaces, furent colorées en vermillon ${ }^{48}$. L'artifice est ici justifié par l'iconographie et la référence antique : "rien ne marque mieux la pudeur que le voile \& le vermillon. Ce dernier prouve même que c'était une divinité. Les anciens avaient accoutumé de peindre avec cette couleur le visage de leurs dieux ${ }^{49} »$. L'auteur livre ensuite la recette employée :

"Au reste, on voit par ce que je viens de dire, que je ne suis pas du sentiment de ceux qui croient que le vermillon des joues de cette statue est naturel au marbre, très certainement, il est ajouté. Le Père Kirker [sic] a parlé fort au long dans un de ses ouvrages (Mundus subterraneus), de l'art de faire pénétrer le marbre par la couleur, \& depuis lui, le Père Baldigiani a découvert une manière encore plus facile \& plus simple que la sienne. On prend du sang de dragon en larmes pour la couleur rouge, de la gomme gutte pour le jaune, \& de la gomme dont les Momies sont remplies, pour le noir. On réduit séparément ces gommes en poudre très subtile, on les détrempe ensuite sur le marbre avec d'excellente eau de vie, \& avant 
que d'appliquer la couleur, on fait chauffer le marbre autant qu'il est possible afin qu'elle ait plus de facilité à pénétrer toute la substance ${ }^{50}$. »

Quelque véridique que soit cet " art de faire pénétrer le marbre par la couleur ", plusieurs sources et de caractères très divers, l'une scientifique et l'autre littéraire, attestent de techniques permettant de colorer le marbre. Le Journal économique d'avril 1758 livrait ainsi la Méthode pour préparer une liqueur qui pénètre dans l'intérieur du marbre, de manière qu'on puisse peindre sur la surface des choses qui paraitront aussi en dedans, précisant que cet art avait été pratiqué avec succès par un tailleur de pierres d'Oxford, M. Birde, avant $1660^{51}$. Quant aux Mémoires de l'Académie royale, ils relataient que le comte de Caylus s'était, lui aussi, intéressé au "moyen d'incorporer la couleur dans le marbre \& de fixer le trait ». Il avait assisté aux expériences de M. de La Faye chez " Dropsy, marbrier de Paris ", et constaté que ces dernières " consistaient en deux tranches de marbre blanc, taillées pour servir de tables; \& sur lesquelles on a peint des taches, pour imiter une brèche dont la Nature n'a jamais montré d'exemple; elles représentent alternativement des couleurs d'un gris tirant sur le fer ou l'ardoise, \& d'un jaune foncé \& sourd : les traits qui séparent ces deux couleurs sont d'un rouge brun ». Un autre marbrier, rapporte le même Mémoire, "qui loge sur le rempart », employait de " fausses couleurs » pour " fabriquer des tables chargées de fleurs ${ }^{52} »$. Ces essais ne semblèrent cependant pas satisfaisants à Caylus qui conclut que « toutes les opérations de ce genre qui ont été citées \& rapportées n'ont été qu'une incorporation vague ${ }^{53} »$.

\section{Conclusion}

Ainsi donc, les " marbres " du $\mathrm{xvIII}^{\mathrm{e}}$ siècle, qui n'en sont pas toujours pour les géologues, sont-ils appréciés avant tout en raison de l'heureuse diversité, qui célèbre la beauté de la matière, de leurs veinages, de leurs " figures", et de leurs couleurs. Jaspes, agates, cornalines, brèches, poudingues, coquilliers, marbres composent un nuancier qui fait le bonheur du géologue comme celui de l'amateur (illustration 24, cahier couleur). Nous n'avons envisagé ici ces « heureuses curiosités de la nature » que sont les pierres dures et les marbres que pour leurs veines et leurs couleurs. Bien

des aspects mériteraient encore d'être développés et les champs de la recherche sont, en ce domaine, importants. L'étude du goût pour les pierres "susceptibles de prendre un beau poli » trouverait sans doute des prolongements savoureux dans celle de leur imitation. Mais même très incomplet, ce bref aperçu du goût pour les marbres colorés et veinés démontre que leur appréciation esthétique, qui se conjugue parfois avec une véritable recherche scientifique sur la compréhension de leur formation, relève de la " curiosité ", phénomène que historiens des sciences comme Lorraine Daston et 
Katharine Park et des historiens ou historiens de l'art comme Francis Haskell, Krzysztof Pomian, Antoine Schnapper, Arthur MacGregor, Robert John Weston Evans, Alexander Marr et Neil Kenny ${ }^{54}$ étudièrent brillamment pour la période moderne, à un moment où, comme le résume Stéphane Van Damme, " un travail de distinction voit ainsi le jour entre une conception ancienne de la curiosité, portée par le merveilleux et la démarche scientifique $^{55}$ ».

Le nuancier de la nature n'est jamais si beau que lorsqu'il est mis en valeur, accompagné, magnifié par le talent des artistes qui osent s'en emparer; les marbriers qui coupent, tracent, et composent de vastes tableaux colorés, les orfèvres et les bronziers qui montent, comme des pierres précieuses, ces « heureuses curiosités de la nature ». Comme le résume Roger Caillois, poète des pierres, " Nulle régularité en effet dans le dessin des agates. Il semble contenir le répertoire entier, le vacarme et l'opulence des formes libres, telles qu'un jour l'ingéniosité et la fantaisie des hommes inventeront, non sans complaisance, de les multiplier ${ }^{56} »$.

\section{Notes}

1. Cet article est tiré d'une conférence donnée dans le cadre du Festival d'histoire de l'art de Fontainebleau, en mai 2015. La partie " géologique ", qui avait été réalisée par Francis Tourneur, ne sera pas développée, tandis que la partie historique, relevant de l'histoire du goût, est ici reprise en partie. Elle sera plus amplement exposée dans notre ouvrage à paraître chez Arthéna en 2017, Versailles en ses marbres, politique royale et marbriers du Roi.

2. Maître de conférences à l'université de Lille 3, membre du centre de recherche de l'IRHIS et directrice des études à l'École du Louvre, Sophie Mouquin travaille sur les arts décoratifs et sur l'histoire du goût pour le marbre aux $\mathrm{XVII}^{\mathrm{e}}$ et $\mathrm{XVIII}^{\mathrm{e}}$ siècles. Elle est l'auteur de plusieurs ouvrages (dont Pierre IV Migeon, 2001; Le style Louis XV, 2003; Écrire la sculpture, 2011; Versailles en ses marbres, politique royale et marbriers du Roi, à paraître en 2017).

3. À ce sujet, voir entre autres Raniero GNOLI, Marmora romana, Rome, Edizioni dell'Elefante, 1971; Marilda de Nuccio et Lucrezia Ungaro (dir.), I marmi colorati della Roma imperiale, Rome, Marsilio, 2002; Dario Del BufAlo, Marbres de couleur, pierres et architecture de l'Antiquité au XVIII siècle, Arles, Actes Sud, 2004; Lorenzo Lazzarini, Pietre e marmi antichi. Natura, caratterizzazione, origine, storia d'uso, diffusione, collezionismo, Padoue, Cedam, 2004; Patrizio PEnsabene, I marmi nella Roma antica, Rome, Carocci, 2014; Henry William Pullen, Manuale dei marmi romani antichi, tradotto, curato, illustrato e aggiornato da Francesco Crocenzi, Rome, Gangemi editore, 2015.

4. Jacques DUBARRY DE LAssale, Identification des marbres, Turin, H. Vial, 2000; id., Utilisation des marbres, Turin, H. Vial, 2005.

5. Geneviève Bresc-BAutier et Hélène DU MESNIL, « La politique royale du marbre français (1700-1789) ", Colloque international tenu dans le cadre du $108^{e}$ congrès national des sociétés savantes, Grenoble, 5-9 avril 1983, Paris, CTHS, 1986, p. 425442 ; Geneviève BRESC-BAUTIER, « Le marbre du Roi : L'Approvisionnement en marbre des bâtiments du Roi, 1660-1715 », Eighteenth Century Life, vol. 17, n 2, mai 1993, p. 36-54; id., "Les marbres des Pyrénées sous Louis XIV », Les Marbres blancs des Pyrénées approches scientifiques et historiques, Entretiens d'archéologie et d'histoire, Saint-Bertrand-de-Cominges, Toulouse, 1995, p. 261-273; id., " L'importation du marbre de Carrare à la cour de Louis XIV, rivalités des marchands et échecs des compagnies ", dans Pascal Julien (dir.), Marbres de Rois, Aix-enProvence, Publications de l'université de Provence, 2013, p. 123-150.

6. Jean-Louis Bonnet, « Des carrières aux marbriers de Caunes-Minervois (xvII ${ }^{\mathrm{e}}$ siècle) ", Bulletin de la Société d'Études Scientifiques de l'Aude, xcvin, 1998, p. 89-102; Pascal Julien, « Le flottage des marbres royaux des Pyrénées à l'Océan ", Forêts et transport. Les modes traditionnels, Paris, ENS, Cahiers d'Études, 14, 2004, p. 25-29; id., " Le Mémoire sur les marbres de Marc-François de Lassus description inédite des Pyrénées au xvIII ${ }^{\mathrm{e}}$ siècle ", Revue de Comminges et des Pyrénées centrales, cxx, avril juin 2004, p. 197-234; id., Marbres, de carrières en Palais, Manosque, Le Bec en l'Air, 2006; Jean-Louis Bonnet et Pascal Julien, «Un temporel de marbre : les carrières de l'abbaye de Caunes-Minervois, aux $\mathrm{XVII}^{\mathrm{e}}$ et $\mathrm{XVIII}^{\mathrm{e}}$ siècles ", Le village et l'abbaye de Caunes-Minervois, Caunes, novembre 2003, Archéologie du Midi Médiéval, 6, 2010, p. 143-151; Pascal Julien, « Des "plaies infli- 
gées aux montagnes" : carrières de marbre des Pyrénées, Minervois et Provence, $\mathrm{XVI}^{\mathrm{e}}$-xIX ${ }^{\mathrm{e}}$ siècles ", Une longue histoire. La construction des paysages méridionaux, 2012, p. 43-55; id. (dir.), Marbres de Rois, Aix-en-Provence, Publications de l'université de Provence, 2013.

7. Notamment : Sophie MouQuin, « La dynastie Derbais : des marbriers brabançonnais au service du roi de France ", Pouvoir(s) de marbres, Liège, 2004, p. $96-108$; id., « Lorsque les marbriers de Versailles venaient du Bas-Hainaut », Société d'Histoire régionale de Rance, 2005, p. 147-178; id., "Versailles en ses marbres, étude d'un décor marmoréen du Grand siècle : l'appartement des Bains ", Revue de l'Art, 2006, n 151, p. 51-64; id., "Versailles, un édifice de marbre : le rouge de Rance et les harmonies colorées versaillaises ", Les Wallons à Versailles, Liège, La Renaissance du Livre, 2007, p. 355-388; id., " Deux projets marbriers abandonnés : la cinquième chapelle de Versailles et l'église royale des Invalides », Livraisons d'histoire de l'architecture, "Grands chantiers et matériaux ", n ${ }^{\circ} 16,2008$, p. $47-57$; id., « Marbres et marbriers de la Galerie des Glaces ", La galerie des Glaces après sa restauration: contexte et restitution, École du Louvre, Paris, 2013, p. 74-96; id., "Les marbriers du Roi, organisation et réalisations ", dans Pascal Julien (dir.), Marbres de Rois, Aix-en-Provence, Publications de l'université de Provence, 2013, p. 203-213; id., Versailles en ses marbres, politique royale et marbriers $d u$ Roi, Paris, Arthéna, à paraître (2017).

8. Alexandre Cojannot, "Mazarin et le "grand dessein" du Louvre, projets et réalisations de 1662 à 1664 ", Bibliothèque de l'École des chartes, $\mathrm{n}^{\circ}$ 161, 2003, p. 133-219; id., " À l'origine de l'architecture de marbre sous Louis XIV : les projets de Louis Le Vau pour le collège Mazarin, le Louvre et Versailles (1662-1663) ", Revue de l'art, n 169, 2010-3, p. 11-23; Muriel BARBIER, "L'architecte, le sculpteur, le marbrier et quelques cheminées bellifontaines des années 1730-1740 », Histoire de l'art, n ${ }^{\circ}$ 57, 2005, p. 67-77.

9. Voir, en plus des ouvrages concernant l'Italie, précédemment cités, entre autres : Nicoletta MoRello, La machina della terra, Teorie geologiche dal Seicento all'Ottocento, Turin, Loescher, 1979; Valentina Gagliardo Bruccia et Giuseppe Montana, I marmi e $i$ diaspri del barocco siciliano, Palerme, Flaccovio, 1998; Monica T. Price, Decorative stones, the complete sourcebook, Londres, Thames \& Hudson, 2007; Sophie Mouquin, " D'agate, de jaspe et de sardoine : pierres fines dans les collections minéralogiques françaises au XVIII ${ }^{\mathrm{e}}$ siècle ", Le luxe, le goût, la science, Neuber, orfêvre minéralogiste à la cour de Saxe, Saint-Rémy-en-l'eau, Monelle Hayot, 2012, p. 45-90; id., " Pour Dieu et pour le
Roi : l'élaboration d'une symbolique du marbre sous l'Ancien Régime », Marbres jaspés de Saint-Rémy et de la région de Rochefort, Namur, TreMa, 2012, p. 205-231.

10. Voir notamment Geneviève BRESC-BAUTIER, "Catherine de Médicis : la passion du marbre ", dans Sabine Frommel et Gerhard Wolf (dir.), Il Mecenatismo di Caterina de'Medici, Padoue, Marsilio, 2008, p. 251-277; Francis TourneuR, « La polychromie des châteaux renaissants : le cas du pavillon d'Anet ", dans Jacques Toussaint (éd.) Rouges et noirs, Rubis, grenat, onyx, obsidienne et autres minéraux rouges \& noirs dans l'art et l'archéologie, Namur, coll. Monographie du TreM.a, 2014, p. $59-63 ; i d$. , " "La nature admirable des pierres sous diverses couleurs et qualitez" : de l'emploi du marbre dans l'architecture de Philibert De l'Orme", dans Frédérique Lemerle et Yves Pauwels (dir.), Philibert De l'Orme. Un architecte dans l'histoire, Arts. Sciences. Techniques, Turnhout, Brepols, 2016, p. 137-150.

11. Antoine Furetière, Dictionnaire universel : contenant generalement tous les mots françois, tant vieux que modernes, E les termes de toutes les sciences et des arts..., 3 vol., La Haye et Rotterdam, A. et R. Leers, 1690, II, p. 552.

12. CEuvres complètes de Stace, traduites par MM. Rinn et Achaintre, 4 vol., Paris, Panckoucke, 1829-1832, I, 1829, note n ${ }^{\circ} 12$, p. 64-65; II, 1829, p. 57.

13. Pline L'AnCien, Histoire naturelle de Pline traduite en François avec le texte latin rétabli d'après les meilleures leçons manuscrites, 12 vol., Paris, veuve Desaint, 1771-1782, XI, 1778, p. 379.

14. François DE Salignac DE La Mothe, Démonstration de l'existence de Dieu, tirée de la connoissance de la nature et proportionnée à la faible intelligence des plus simples, Paris, Jacques Estienne, 1713, p. 12-13.

15. Gottfried Wilhelm LeIBnIz, Nouveaux essais sur l'entendement humain, Paris, 1765, rééd., Paris, Flammarion, 2002, p. 36-37.

16. Gilles Deleuze, Le Pli, Leibniz et le Baroque, Paris, Éditions de Minuit, 1988, p. 5-6, cité par Mireille BuydENS, " La forme dévorée, pour une approche Deleuzienne d'Internet », dans Thierry Lenain (dir.), Deleuze, Foucault, Lyotard, Paris, Vrin, 1997, p. 46.

17. Fabio BARRY «Walking on water : cosmic floors in antiquity and in the middle ages ", The Art Bulletin, vol. LXxxIx, $\mathrm{n}^{\circ}$ 4, décembre 2007, p. 627-656.

18. Traduction de l'auteur " Le naturali vene e macchie del marmo possono no di rado combinarsi in modo da simulare o suggerire immagini di varia specie. Questo succede in special modo nella decorazione parietale costituita da lastroni di marmo segati dallo stesso blocco e quindi, come di dice, 
messi in opera ad apertura ». R. GNOLI, Marmora romana, op. cit., p. 53.

19. Jean D'Ormesson, La gloire de l'Empire, Paris, Gallimard, 1971, p. 25.

20. André BRETON, «Langue des pierres », Le surréalisme, $\mathrm{n}^{\circ} 3$, automne 1957, p. 64-68; id., Perspective cavalière, Paris, Gallimard, 1970; Roger CAILlois, Pierres, suivi d'autres textes, Paris, Gallimard, 1966, rééd., 2013; id., L'écriture des pierres, Paris, Skira, 1971, rééd., Paris, Flammarion, 1981; Jurgis Baltrušaitis, Aberrations, quatre essais sur la légende des formes, Paris, Perrin, 1957.

21. Sur ce sujet, récemment célébré dans une exposition au musée Guimet (Catherine Delacour (dir.), Rochers de lettrés, itinéraires de l'art en Chine, Paris, RMN, 2012), voir notamment R. CAIllois, Pierres, op. cit., 1971, rééd., 1981; Olivier ScHEFER, " Les pierres de rêve : minéralogie visionnaire ", dans Vincent Gille (dir.), Trajectoires $d u$ rêve, $d u$ romantisme au surréalisme, Paris, Pavillon des arts, 2003, p. 203-210; Victoria CIRLOT, "Langue des pierres : expérience mystique et nature », dans Dominique de Courcelles (dir.), Les enjeux philosophiques de la mystique, Jérôme Grenoble, Millon, 2007, p. 71-90.

22. La bibliographie, sur ce sujet, est abondante. Voir notamment Annamaria GIUsti, Tesori di pietre dure : Palazzo Pitti, Uffizi e altri luoghi d'arte a Firenze, Milan, Electa, 1989; id., Pietre Dure. Hardstone in furniture and decorations, Londres, Ph. Wilson, 1992; Adalgisa LugLI, Naturalia et mirabilia : les cabinets de curiosités en Europe, Paris, Adam Biro, 1998; Daniel Alcouffe, La collection de gemmes de Louis XIV, Paris, RMN, 2001; Lorraine DASTON et Katharine PARK, Wonders and the Order of Nature (1150-1750), New York, Zone Books, 1998, rééd. 2001; Stéphane CAstelluccio, Les collections royales d'objets d'art, de François I ${ }^{e r}$ à la Révolution, Paris, L'Amateur, 2002; Annamaria GIUsTI, Eternità e nobiltà di materia : itinerario artistico fra le pietre policrome, Florence, Polistampa, 2003; Robert John Weston Evans et Alexander Marr, Curiosity and Wonder from the Renaissance to the Enlightenment, Ashgate, Aldershot, 2006; Wolfram Koeppe et Annamaria Giusti, Art of the Royal Court: Treasures in Pietre Dure from the Palaces of Europe, New Haven, Yale University Press, 2008; Paola Granata (dir.), Dal libro di natura al teatro del mondo : studi in onore di Adalgisa Lugli, Bologne, Fausto Lupetti, 2012. Sophie MouQuIN, "Entre curiosité et science : lithothèques et marmothèques sous l'Ancien Régime ", Studiolo, $\mathrm{n}^{\circ}$ 9, L'œuvre et sa présentation, 2012, p. 74-98. Sophie Mouquin, " D'agate, de jaspe et de sardoine ", op. cit., p. 45-90.

23. Cité dans V. Cirlot, "Langue des pierres", op. cit., 2007, p. 75 .
24. Jurgis BALtrušAitis, Aberrations, quatre essais sur la légende des formes, Paris, Perrin, 1957, p. 57.

25. Jacques-Christophe VAlmont DE Bomare, Dictionnaire raisonné universel d'Histoire naturelle, 6 vol., Paris, Brunet, 1767-1768, II, p. 23.

26. Joscelyn Godwin, Athanasius Kircher, Le Théâtre du Monde, trad. fr. par Charles Moysan, Paris, Imprimerie nationale, 2009, p. 148.

27. Élie Bertrand, Mémoires sur la structure intérieure de la terre, Zurich, Heidegguer et compagnie, 1752 , p. 46.

28. Benoît DE MAILlET, Telliamed ou entretiens d'un philosophe indien avec un missionnaire français sur la diminution de la mer, la formation de la terre, l'origine de l'homme, Ec, 2 vol., Amsterdam, L'Honoré \& Fils, 1748, I, p. 53

29. Georges-Louis Leclerc de Buffon, Histoire naturelle des minéraux, 5 vol., Paris, Imprimerie royale, $1783-1788, \mathrm{I}, 1783$, p. 310.

30. Ibid., p. 311.

31. Antoine-Joseph Dezallier d'Argenville, Histoire naturelle éclaircie dans une de ses parties principales, l'oryctologie qui traite des terres, des pierres, des métaux, des minéraux et autres fossiles, ouvrage dans lequel on trouve une nouvelle méthode latine et française de les diviser, E une notice critique des principaux ouvrages qui ont paru sur ces matières, Paris, De Bure l'aîné, 1755, p. 205.

32. Journal économique, juin 1759, p. 241.

33. Antoine-Joseph Dezallier D'Argenville, L'Histoire naturelle éclaircie dans deux de ses parties principales. La lithologie et la conchyliologie dont l'une traite des pierres et l'autre des coquillages, Paris, 1742, p. 59 et id., Histoire naturelle, op. cit., 1755, p. 205. Il reprenait vraisemblablement Bernard Palissy (voir Bernard PALISsy, Discours admirables de la nature des eaux et fontaines, tant naturelles qu'artificielles, des métaux, des sels et salines, des pierres, des terres $d u$ feu et des émaux, Paris, Martin le jeune, 1580, p. 242 et $i d$., CEuvres de Bernard Palissy, revues sur les exemplaires de la bibliothèque du Roi, avec des notes, par M. Fauvas de Saint-Fond, et des additions par M. Gobert, Paris, Ruault, 1777, p. 124).

34. B. DE MAILlet, Telliamed, op. cit., p. 45-46.

35. Ibid., I, p. 46.

36. Ibid., p. 56.

37. Nicolas LÉmery, Traité universel des drogues simples mises en ordre alphabétiques, Paris, Laurent d'Houry, 1698, 2e éd., Paris, Laurent d'Houry, 1714, p. 528.

38. Michel-Jean DE Borch, Minéralogie sicilienne docimastique et métallurgique ou connaissance de tous les minéraux que produit l'ile de Sicile, avec les détails des mines et des carrières et l'histoire des travaux anciens et actuels de ce pays, Turin, frères Reycends, 1780, p. 100-106. 
39. Georges-Louis LeCLERC de Buffon, Histoire naturelle des minéraux, 5 vol., Paris, Imprimerie royale, 1783-1788, I, 1783, p. 301.

40. Ibid., p. 309.

41. Pour la période antique, voir notamment : Max Schvoerer, Archéomatériaux. Marbres et autres roches, $4^{e}$ Conférence internationale de l'Association pour l'étude des marbres et autres roches utilisés dans le passé, ASMOSIA 4, Bordeaux, Presses Universitaires de Bordeaux, 1995; Brigitte Bourgeors et Philippe JOCKEY, « Approches nouvelles de la polychromie des sculptures hellénistiques de Délos ", Comptes rendus des séances de l'Académie des Inscriptions et Belles Lettres, 2001, vol. 145, $\mathrm{n}^{\circ}$ 1, p. 629-665; Michales A. Tiverios et Despoina S. Tsiafakis (dir.), Color in Ancient Greece. The Role of Color in Ancient Greek Art and Architecture 700-31 B.C., Thessalonique, 2002; Sandrine Dubel, Valérie NaAs et Agnès Rouveret, (dir.), Couleurs et matières dans l'Antiquité, textes, techniques et pratiques, Paris, Éditions rue d'Ulm, 2006; Sophie Descamps-Lequime (dir.), Peinture et couleur dans le monde grec antique, Paris, Musée du Louvre, 2007; Roberta Panzanelli (dir.), The Color of Life: Polychromy in Sculpture from Antiquity to the Present, Los Angeles, The J. Paul Getty Museum, 2008; Adeline Grand-Clément, " Les marbres antiques retrouvent des couleurs : apport des recherches récentes et débats en cours ", Anabases, 10 (2009), p. 243-250.

42. Pline l'ANCIEN, op. cit., p. 161.

43. À ce sujet, voir notamment les publications de Joscelyn Godwin et Paula FindLen : J. Godwin, Athanasius Kircher, un homme de la Renaissance à la quête du savoir perdu (1979), trad. fr. Paris, B. Diffusion, 1980; id., Athanasius Kircher, Le Thêâtre $d u$ Monde, trad. fr. Paris, Imprimerie Nationale, 2009; P. FindLEn, Athanasius Kircher : the last man who knew everything, New York, Routledge, 2004.

44. Nicolas LÉMERY, Recueil de curiositez rares et nouvelles dans les plus admirables effets de la Nature, Lausanne, David Gentil, 1681, p. 57-58.

45. Histoire l'Académie royale des sciences, 1730, p. 50.

46. M.-J. DE Borch, Minéralogie sicilienne, op. cit., p. 230.

47. Alexis-Frederik Cronstedt, Essai d'une nouvelle minéralogie. Tr. du suédois \& de l'allemand de M. Wiedman Ec. Ec., par M. Dreux fils, Paris, Didot, 1771, p. 56.
48. La Pudicité, marbre, $198 \times 59 \times 49$ cm, Versailles, châteaux de Versailles et Trianon, Grands Appartements, galerie des Glaces, inv. MR245. Statue antique provenant de Benghazi, placée dans la galerie des Glaces en 1695 (remise à son emplacement d'origine en 1948, dépôt du Musée du Louvre).

49. Jean-Aymar Piganiol de La Force, Nouvelle Description des châteaux et parcs de Versailles et de Marly contenant une explication historique de toutes les Peintures, Tableaux, Statues, Vases \& Ornemens qui s'y voyent, leurs dimensions, $\mathcal{E}$ les noms des Peintres, des Sculpteurs $\&$ des Graveurs qui les ont faits, Paris, F. et P. Delaulne, 1701, 9e éd., 2 vol., Paris, Hocherau, 1764, p. 218.

50. Ibid., p. 220-221.

51. Journal économique, avril 1758, p. 169.

52. Histoire de l'Académie royale des Inscriptions \& Belles Lettres, XIV, 1772, p. 304.

53. Ibid., p. 297-298.

54. L. DAston et K. PARK, Wonders, op. cit., 1998, rééd. 2001; Lorraine Daston, Natural Law and Laws of Nature in Early Modern Europe, Ashgate, Aldershot, 2008; Francis Haskell, De l'art et du goût, jadis et naguère, Paris, Gallimard, 1989; Krzysztof Pomian, Collectionneurs, amateurs et curieux, Paris, Venise, XVIXVIII siècle, Paris, Gallimard, coll. "Bibliothèque des histoires ", 1987; Antoine SCHNAPPER, Le Géant, la licorne et la tulipe, collections et collectionneurs dans la France $d u$ XVIII ${ }^{e}$ I, Histoire et histoire naturelle, Paris, Flammarion, 1988; id., Curieux du grand siècle, collections et collectionneurs dans la France $d u$ XVII siècle, II, CEuvres d'art, Paris, Flammarion, 1994; Arthur MacGregor, Curiosity and Enlightenment, Collectors and Collections from the Sixteenth to the Nineteenth Century, New Haven, Yale University Press, 2007; R.J. Weston Evans et A. Marr, Curiosity, op. cit., 2006; Neil KenNy, Curiosity in Early Modern Europe: Word Histories, Harrassowitz Verlag, Wolfenbütteler Forschungen, 1998; id., The Uses of Curiosity in Early Modern France and Germany, Oxford, Oxford University Press, 2004.

55. Stéphane VAN Damme, « La curiosité histoire d'un mot ", dans Dominique Pestre (dir.), Histoire des sciences et des savoirs, 1; De la Renaissance aux Lumières, Paris, Le Seuil, 2015, p. 136-137.

56. R. CAillois, Pierres, op. cit., 1966, rééd. 2013, p. 41. 